\title{
Endovascular thrombectomy in acute ischemic stroke
}

\author{
Raed A. Joundi MD DPhil, Karl Boyle MD MSc
}

\section{Endovascular thrombectomy with retrievable stents is the standard of care for patients with acute ischemic stroke from large-vessel occlusion}

Five recent well-designed randomized controlled trials showed improved outcomes with combined endovascular thrombectomy and intravenous thrombolysis compared with thrombolysis alone. ${ }^{1}$ Recent guidelines ${ }^{2,3}$ recommend endovascular thrombectomy for patients with large-vessel intracranial occlusion (i.e., occlusion of the distal internal carotid artery or proximal middle cerebral artery) identified on vascular imaging, in conjunction with substantial neurologic deficits, lack of an established infarct (i.e., a small core) and the ability to provide rapid treatment with groin puncture occurring within six hours of symptom onset.
Endovascular thrombectomy with retrievable stents is safe

Adverse effects with endovascular thrombectomy are minimal, with low rates of procedural complications and no increase in symptomatic intracranial hemorrhage, despite use of thrombolysis. ${ }^{1}$ Distal embolization occurs in about $5 \%$ of cases. ${ }^{5}$

\section{As with intravenous thrombolysis, a rapid workflow is critical}

In one of the trials of endovascular thrombectomy, patients received treatment up to 12 hours from symptom onset. ${ }^{1}$ However, the longest median time from symptom onset to treatment in any of the trials was five hours, and aggressive time-based treatment targets were established, ${ }^{1}$ such as 60 minutes for imaging-to-groin puncture and 90 minutes for imaging-to-reperfusion. ${ }^{4}$ Endovascular thrombectomy with
retrievable stents is highly effective

All five trials consistently showed a substantial positive benefit, ranging from $14 \%$ to $31 \%$ absolute difference in favour of the intervention in achieving a score of $0-2$ on the modified Rankin Scale (alive and independent) at 90 days (Appendix 1, available at www.cmaj.ca/ lookup/suppl/doi:10.1503/cmaj.150875 /-/DC1). ${ }^{1}$ In the only Canadian trial, the number needed to treat for independence was 4, and mortality was halved from $19 \%$ to $10 \%$. $^{4}$

\section{Thrombolysis remains essential}

Patients who are potentially eligible for endovascular therapy and intravenous thrombolysis (i.e., within four and a half hours of symptom onset) must still receive intravenous thrombolysis before the procedure. Noninvasive vascular imaging should immediately follow noncontrast computed tomography to ensure timely selection of patients, but should not delay treatment with intravenous thrombolysis. ${ }^{2}$

Competing interests: None declared.

3. Casaubon LK, Boulanger J-M, Blacquiere D, et al.; Heart and Stroke Foundation of Canada Canadian Stroke Best Practices Advisory Committee. Canadian stroke best practice recommendations: hyperacute stroke care guidelines, update 2015. Int J Stroke 2015;10:924-40.

4. Goyal M, Demchuk AM, Menon BK, et al.; ESCAPE Trial Investigators. Randomized assessment of rapid endovascular treatment of ischemic stroke. N Engl J Med 2015;372:1019-30.

5. Berkhemer OA, Fransen PSS, Beumer D, et al. A randomized trial of intraarterial treatment for acute ischemic stroke. N Engl J Med 2015;372:11-20.
This article has been peer reviewed.

Affiliations: Division of Neurology (Joundi, Boyle), Department of Medicine, University of Toronto; Regional Stroke Centre (Boyle), Sunnybrook Health Sciences Centre, Toronto, Ont.

Correspondence to: Karl Boyle, karl.boyle@ sunnybrook.ca

CMAJ 2016. DOI:10.1503/cmaj.150875 